\title{
ERROS DE MEDICAÇÃO E GRAU DE DANO AO PACIENTE EM HOSPITAL ESCOLA
}

\author{
Miriam Cristina Borges ${ }^{1}$, Josimerci Ittavo Lamana Faria ${ }^{2}$, Maria Regina Lourenço Jabur ${ }^{3}$, Kleber Aparecido de \\ Oliveira $^{4}$, Ilza dos Passos Zborowski ${ }^{3}$, Lúcia Marinilza Beccaria ${ }^{2}$
}

\begin{abstract}
RESUMO: O estudo teve como objetivos verificar a incidência de erros e quase erros de medicação e grau de dano ao paciente, e a associação entre o grau de dano e características sociodemográficas e da internação. Estudo descritivo, retrospectivo, com abordagem quantitativa, realizado em hospital no interior do estado de São Paulo. Os dados foram coletados por meio do sistema de notificação de eventos adversos e prontuário eletrônico, entre setembro/2014 e agosto/2015. Verificou-se 113 notificações de erros, a maioria relacionada ao preparo e administração; o quase erro apresentou pequena porcentagem; os danos foram classificados: 60 (53,1\%) nenhum, 29 (25,7\%) leve, $16(14,1 \%)$ moderado e oito $(7,1 \%)$ grave. Houve maior notificação em unidades clínico-cirúrgicas, e evidenciou-se relação entre gravidade do evento e pacientes de terapia intensiva. Notificar, mensurar e analisar a ocorrência, o grau de dano e relacionar com as características do paciente auxiliam o enfermeiro a melhorar o processo de trabalho na prevenção e controle dos erros de medicação.
\end{abstract}

DESCRITORES: Segurança do paciente; Erros de medicação; Indicador de qualidade; Notificação; Hospital de ensino.

\section{MEDICATION ERRORS AND DEGREE OF PATIENT DAMAGE AT A TEACHING HOSPITAL}

ABSTRACT: The objectives in this study were to verify the incidence of medication errors and near misses and the degree of damage to the patient, as well as the association between the degree of damage and sociodemographic and hospitalization characteristics. Descriptive and retrospective study with a quantitative approach, undertaken at a hospital in the interior of the State of São Paulo. The data were collected through the adverse event reporting system and the electronic history between September/2014 and August/2015. In total, 113 error reports were found, mostly related to preparation and administration; a small percentage of near misses was found; the damage was classified: 60 (53.1\%) none, $29(25.7 \%)$ mild, $16(14.1 \%)$ moderate and eight $(7.1 \%)$ severe. More errors were reported at clinical-surgical units, and a relation was found between the severity of the event and intensive care patients. Reporting, measuring and analyzing the event, the degree of damage and relating them with the patient characteristics help the nurse to improve the work process in the prevention and control of medication errors.

DESCRIPTORS: Patient safety; Medication errors; Quality indicators; Notification; Hospitals, teaching.

\section{ERRORES DE MEDICACIÓN Y GRADO DE DAÑO AL PACIENTE EN HOSPITAL ESCUELA}

RESUMEN: Los objetivos en este estudio fueron verificar la incidencia de errores y casi-errores de medicación y grado de daño al paciente, y la asociación entre el grado de daño y características sociodemográficas y de la internación. Estudio descriptivo, retrospectivo, con aproximación cuantitativa, desarrollado en un hospital en el interior del estado de São Paulo. Los datos fueron recolectados mediante el sistema de notificación de eventos adversos yarchivo electrónico, entre septiembre/2014 y agosto/2015. Fueron verificadas 113 notificaciones de errores, la mayoría relacionada a la preparación y administración; el porcentaje de casi errores fue pequeño; los daños fueron clasificados: 60 (53,1\%) ningún, 29 (25,7\%) leve, 16 (14,1\%) moderado y ocho (7,1\%) grave. Fue encontrada mayor notificación en unidades clínico-quirúrgicas, y fue evidenciada relación entre gravedad del evento y pacientes de terapia intensiva. Notificar, mensurar y analizar la ocurrencia, el grado de daño y relacionar con las características del paciente ayudan al enfermero a mejorar el proceso de trabajo en la prevención y el control de los errores de medicación.

DESCRIPTORES: Seguridad del paciente; Errores de medicación; Indicadores de calidad; Notificación; Hospitales de enseñanza.

'Enfermeira. Aperfeiçoamento em Educação Permanente e Segurança do Paciente. Fundação Faculdade de Medicina de São José do Rio Preto. São José do Rio Preto, SP, Brasil.

${ }^{2}$ Enfermeira. Doutora em Enfermagem. Docente de Enfermagem da Faculdade de Medicina de São José do Rio Preto. São José do Rio Preto, SP, Brasil.

${ }^{3}$ Enfermeira. Doutora em Enfermagem. Fundação Faculdade de Medicina de São José do Rio Preto. São José do Rio Preto, SP, Brasil. ${ }^{4}$ Enfermeiro. Mestre em Enfermagem. Fundação Faculdade de Medicina de São José do Rio Preto. São José do Rio Preto, SP, Brasil. 


\section{- INTRODUÇÃO}

Administrar medicamentos é uma das principais atividades da equipe de enfermagem ${ }^{(1)}$. É um processo que envolve etapas de aquisição, armazenamento, prescrição, dispensação, preparo e administração(2). Devido sua complexidade, o erro de medicação pode ocorrer em qualquer uma destas fases, gerando riscos ou até mesmo danos ao paciente ${ }^{(2-3)}$. O monitoramento dos erros de medicação se faz necessário e merece atenção por parte da equipe, portanto, deve ser notificado e mensurado pelo enfermeiro a fim de servir de parâmetro para avaliação da assistência ${ }^{(4)}$.

Estudos realizados em diversas regiões do Brasil demonstraram que a incidência de não conformidades relacionadas à medicação em hospitais gerais, setores de clínica cirúrgica e unidades de terapias intensivas (UTIs) variou de $14,8 \%$ a $24,8 \%{ }^{(5-8)}$, alcançando $82,3 \%$ em uma instituição da rede privada no interior do estado de São Paulo ${ }^{(3)}$. Por outro lado, é importante ressaltar a ocorrência de subnotificações, comprovadas por um estudo realizado no Rio de Janeiro, em que a incidência de erros de medicação representou apenas $4,3 \%$ dos eventos adversos ${ }^{(9)}$.

Aocorrência de erro não é causada somente pelo fator humano, mas também, pelos problemas relacionados ao processo, à sobrecarga de trabalho, déficit de atualização em educação e saúde, manipulação incorreta dos medicamentos, ambiente inadequado ou até mesmo à condição clínica do paciente ${ }^{(10-11)}$.

Na prática assistencial, a administração de medicamentos é uma das atividades de maior relevância, no entanto, observa-se uma tendência da equipe de enfermagem em desconsiderar os erros por acreditar que, na maioria das vezes, não acarretam repercussões mais graves aos pacientes ${ }^{(12)}$.

Considerando a demanda de trabalho advinda do processo de administração de medicamentos, do fato do ser humano não estar isento de erros, assim como a desconsideração dos profissionais de enfermagem quanto aos eventos não graves, os objetivos deste estudo foram verificar a incidência de erros e quase erros de medicação, bem como o grau de dano ao paciente e identificar a associação entre o grau de dano e as características sociodemográficas e da internação.

\section{- MÉTODO}

Estudo descritivo, retrospectivo, com abordagem quantitativa, realizado em um hospital do noroeste paulista, de porte especial, geral, de ensino, com aproximadamente 700 leitos. É uma instituição de nível terciário, credenciada para atendimento de pacientes de alta complexidade, que atende $85 \%$ deles pelo Sistema Único de Saúde (SUS), mas também serviços de saúde suplementar e particulares. Além disso, destaca-se pelas atividades de ensino e pesquisa. Pertence à Rede de Hospitais Sentinela da Agência Nacional de Vigilância Sanitária, cuja finalidade é diagnosticar eventos adversos e queixas técnicas relacionadas a produtos de saúde, por meio do Gerenciamento de Riscos.

Os critérios de inclusão foram pacientes adultos internados, que sofreram alguma não conformidade relacionada à medicação, notificada por meio do sistema informatizado, no período de setembro de 2014 a agosto de 2015.

Os critérios de exclusão foram notificações de erros e quase erros provenientes de outras instituições ligadas ao hospital, cujo monitoramento também é realizado pela Unidade de Gerenciamento de Riscos do hospital estudado; de unidades que não registravam tal indicador, de eventos ocorridos com pacientes ambulatoriais e presença de dados incompletos no sistema de informação do hospital. Seguindo tais critérios, foram excluídas 19 notificações provenientes do setor de recuperação pós-anestésica.

A incidência de erros e quase erros de medicação foi calculada utilizando-se as fórmulas do Manual de Indicadores de Enfermagem do Núcleo de Apoio a Gestão Hospitalar, elaborado em parceria com o Programa de Compromisso com a Qualidade Hospitalar (PCQH). Para os erros de medicação o cálculo realizado foi " $\mathrm{n}$ - de erros relacionados à administração de medicamento/no de pacientes-dia x 100" e para o quase erro, " $\mathrm{n}$ - de quase falhas relacionadas ao processo de administração de medicação/no pacientes-dia x 100".

Os dados foram coletados das fichas de notificação de eventos adversos, preenchidas por profissionais de saúde, por meio do sistema informatizado no período selecionado. Estas fichas abrangem a identificação do 
notificador (nome, profissão, unidade de trabalho e data do cadastro da notificação), data de ocorrência do evento, identificação do paciente (quarto, leito, unidade de internação, nome e prontuário), tipo de evento, descrição e gravidade do dano. É importante destacar que em alguns casos ocorreram mais de um incidente com o mesmo paciente.

Para determinar a gravidade do evento, utilizou-se a Classificação Internacional para a Segurança do Paciente da Organização Mundial da Saúde ${ }^{(13)}$. Além destes dados, foram pesquisadas no prontuário eletrônico do paciente, informações complementares como, idade, raça, escolaridade, procedência, profissão, religião, convênio, número de dias de internação, presença de acompanhante no dia do incidente, transferência de setor no dia do incidente, diagnóstico médico segundo a classificação internacional de doenças (CID-10) e desfecho da internação.

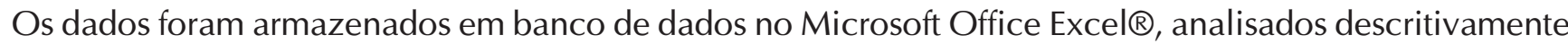
em frequências absolutas e relativas e apresentados em tabelas para melhor visualização. Foi calculado a média e desvio padrão para as variáveis quantitativas (idade e número de dias de internação).

Para análise dos dados foi aplicado teste de correlação de Spearman, por meio do software Prisma versão 6.01, para identificar a relação entre a gravidade do evento e as variáveis sociodemográficas do paciente. Foi adotado um nível de significância de 5\%.

As variáveis testadas foram: sexo, faixa etária, procedência, escolaridade, tipo de convênio, unidade de internação, presença de acompanhante, transferência de unidade no dia do incidente, número de dias de internação, diagnóstico médico e desfecho clínico da internação; selecionadas segundo a relevância para o estudo.

O projeto seguiu todos os preceitos éticos e foi aprovado em 05/05/2015 pelo Comitê de Ética em Pesquisa (CEP) da Faculdade de Medicina de São José do Rio Preto - FAMERP, com o parecer no 1.050.829.

\section{RESUlTADOS}

São apresentados na Tabela 1 indicadores de 113 notificações de erros e quase erros segundo o mês. Nota-se que o quase erro teve incidência bem menor do que os erros e que o indicador de erros apresentou maior valor em maio de 2015 e menor em março de 2015.

Tabela 1 - Incidência de erros e quase erros de medicação. São José do Rio Preto, SP, Brasil, 2016

\begin{tabular}{ccc} 
Período & Indicador Erro & Indicador Quase Erro \\
\hline Sep-14 & 0,09 & - \\
\hline Oct-14 & 0,08 & 0,005 \\
\hline Nov-14 & 0,05 & - \\
\hline Dec-14 & 0,04 & 0,008 \\
\hline Jan-15 & 0,09 & 0,008 \\
\hline Feb-15 & 0,04 & - \\
\hline Mar-15 & 0,007 & - \\
\hline Apr-15 & 0,1 & - \\
\hline May-15 & 0,12 & - \\
\hline Jun-15 & 0,05 & 0,007 \\
\hline Jul-15 & 0,02 & -
\end{tabular}

Dada à baixa frequência do quase erro, a caracterização dos pacientes foi realizada considerando os erros de medicação. A caracterização sociodemográfica e dados relacionados ao período da internação dos pacientes estão apresentados nas Tabelas 2 e 3, respectivamente, assim como os resultados dos testes de associação entre cada uma das variáveis com a gravidade do evento. 
Tabela 2 - Caracterização sociodemográfica. São José do Rio Preto, SP, Brasil, 2016

\begin{tabular}{|c|c|c|}
\hline Dados sociodemográficos & $\mathrm{n}(\%)$ & $p+$ \\
\hline Sexo & & - \\
\hline Masculino & $65(57,6)$ & - \\
\hline Feminino & $48(42,4)$ & 0,432 \\
\hline Faixa etária & Média: 59/ DP 18,19 & - \\
\hline$<40$ anos & $19(16,8)$ & - \\
\hline 41 a 60 anos & $36(31,9)$ & - \\
\hline 61 a 80 anos & $49(43,4)$ & - \\
\hline$>80$ anos & $9(7,9)$ & 0,439 \\
\hline Raça & & - \\
\hline Branca & $99(87,6)$ & - \\
\hline Negra & $7(6,2)$ & - \\
\hline Parda & $3(2,7)$ & - \\
\hline Sem informação & $4(3,5)$ & - \\
\hline Escolaridade & & - \\
\hline Até Ensino Fundamental Incompleto & $72(63,7)$ & 0,668 \\
\hline Até Ensino Médio Incompleto & $7(6,2)$ & - \\
\hline Até Ensino Superior Incompleto & $21(18,6)$ & - \\
\hline Até especialização & $6(5,3)$ & - \\
\hline Sem informação & $7(6,2)$ & - \\
\hline Profissão & & - \\
\hline Aposentados/Pensionistas & $53(46,9)$ & - \\
\hline Atividade remunerada & $34(30,1)$ & - \\
\hline Do lar & $14(12,4)$ & - \\
\hline Estudante & $4(3,5)$ & - \\
\hline Sem informação & $8(7,1)$ & - \\
\hline Religião & & - \\
\hline Católica & $84(74,3)$ & - \\
\hline Evangélica/Pentecostal & $13(11,5)$ & - \\
\hline Outras & $7(6,2)$ & - \\
\hline Nenhuma & $1(0,9)$ & - \\
\hline Sem informação & $8(7,1)$ & - \\
\hline Procedência & & - \\
\hline São José do Rio Preto & $43(38,1)$ & 0,861 \\
\hline Região de São José do Rio Preto & $67(59,3)$ & - \\
\hline Outro estado & $3(2,6)$ & - \\
\hline Total & 113(100) & - \\
\hline
\end{tabular}

+ Teste de Spearman: associação entre a gravidade do dano e os dados sociodemográficos

Tabela 2 - Caracterização sociodemográfica. São José do Rio Preto, SP, Brasil, 2016 (continua)

\begin{tabular}{lcc} 
Internação & $\mathbf{n}(\%)$ & $\mathbf{p} \neq$ \\
\hline Convênio & & - \\
\hline SUS & $100(88,5)$ & - \\
\hline Saúde Suplementar & $13(11,5)$ & 0,043 \\
\hline Unidade de Internação & & - \\
\hline
\end{tabular}




\begin{tabular}{lcc}
\hline Unidades abertas & $70(62)$ & - \\
\hline Unidades fechadas & $43(38)$ & 0,001 \\
\hline Diagnóstico Médico & & - \\
\hline Grupo $1 \S$ & $48(42,5)$ & 0,016 \\
\hline Grupo $2+\dagger$ & $38(33,6)$ & - \\
\hline Grupo $3 \neq \neq$ & $19(16,8)$ & - \\
\hline Grupo $4 \S \S$ & $8(7,1)$ & - \\
\hline Dias de internação & Média: $28 /$ DP: 27,76 & \\
\hline Até 7 dias de internação & $22(19,5)$ & - \\
\hline 8 a 15 dias de internação & $20(17,7)$ & - \\
\hline 16 a 30 dias de internação & $36(31,8)$ & - \\
\hline$>30$ dias de internação & $35(31)$ & 0,024 \\
\hline Acompanhante no dia do incidente & & - \\
\hline Não & $59(52,2)$ & 0,057 \\
\hline Sim & $51(45,1)$ & - \\
\hline Sem informação & $3(2,7)$ & - \\
\hline Transferência no dia do incidente & & - \\
\hline Não & $100(88,5)$ & 0,523 \\
\hline Sim & $13(11,5)$ & - \\
\hline Desfecho da internação & & - \\
\hline Alta & $76(67,3)$ & 0,006 \\
\hline Óbito & $37(32,7)$ & - \\
\hline Total & $113(100)$ & - \\
\hline
\end{tabular}

‡ Teste de Spearman: associação entre a gravidade do dano e as características da internação.

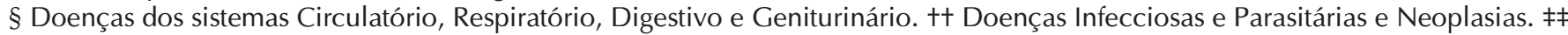
Achados anormais de exames laboratoriais, causas externas ou consequência destas e fatores que influenciam o estado de saúde. $\S \S$ Outros (doenças do sistema nervoso; doenças do sangue; doenças do sistema endócrino; transtornos mentais; doenças do olho e anexos; doenças osteomusculares e do tecido conjuntivo; gestação, parto e puerpério).

A análise de correlação de Spearman mostrou que houve correlação significativa entre os incidentes que ocasionaram maior gravidade e os pacientes atendidos pela saúde suplementar $(p=0,0431)$, hospitalizados em unidades fechadas ( $p=0,0012)$, com período de internação prolongado $(p=0,0238)$ e seu desfecho, $a$ alta hospitalar $(p=0,0061)$.

Quanto à caracterização do incidente notificado, o tipo de erro de medicação e o grau de dano causado encontram-se apresentados na Tabela 4.

Tabela 4 - Caracterização do incidente. São José do Rio Preto, SP, Brasil, 2016

\begin{tabular}{lc} 
Incidente & $\mathbf{n}(\%)$ \\
\hline Tipo de erro & \\
\hline Relacionado aos cinco certos da medicação & $58(51,3)$ \\
\hline Medicamento não administrado & $32(28,3)$ \\
\hline Outros & $23(20,4)$ \\
\hline Grau de dano & \\
\hline Nenhum & $60(53,1)$ \\
\hline Leve & $29(25,7)$ \\
\hline Moderado & $16(14,1)$ \\
\hline Grave & $8(7,1)$ \\
\hline Total & $113(100)$
\end{tabular}


A notificação foi realizada pelo enfermeiro em $99,1 \%$ dos casos e a data da notificação foi diferente do dia que o evento ocorreu em $62,4 \%$, pois o sistema permite realização de notificações retroativas.

\section{DISCUSSÃO}

Estima-se que a ocorrência de eventos adversos em hospitais ocorra em torno de $10 \%$ dos pacientes internados ${ }^{(14-15)}$ entretanto, estudos apontam que esse percentual pode variar de $5 \%(9,16)$ a $41 \%{ }^{(16)}$. Os incidentes sem dano representaram $82 \%$ dos eventos em um estudo realizado no centro-oeste do Brasil ${ }^{(6)}$.

Dentre os possíveis eventos adversos, os erros de medicação, apontados em diversos estudos, têm percentuais variando de $3 \%$ a $54 \%^{(2,5-7,9,17-18)}$. É importante destacar que, assim como os demais eventos adversos, eles devem ser notificados, pois só assim poderão gerar indicadores de qualidade na assistência ${ }^{(19)}$.

Constatou-se que a maioria dos pacientes que tiveram algum incidente notificado, relacionado à medicação durante sua hospitalização, eram do sexo masculino, o que corrobora com outros estudos ${ }^{(12,18)}$. A literatura aponta que o sexo masculino é mais frequentemente acometido em relação aos eventos adversos em geral ${ }^{(5,9)}$. A maior parte dos pacientes era idosa, com idade entre 61 e 80 anos, representando 43,4\% (média: 59/ DP 18,19) $)^{(5,9,12)}$.

Quanto à escolaridade, a maior parte não possuía o ensino fundamental completo, somando-se, inclusive aos não alfabetizados. A maioria estava internada em unidades de clínica médico-cirúrgica ${ }^{(3)}$, de acordo com o perfil da instituição.

O diagnóstico médico da internação para 41,9\% dos pacientes eram Doenças do Sistema Circulatório, Respiratório, Digestivo e Geniturinário, seguido por 33,4\%, que representou as Doenças Infecciosas e Parasitárias e Neoplasias corroborando com achados de outro estudo realizado em instituição acreditada do estado de São Paulo(5).

O tempo médio de internação foi de 28 dias, sendo que 72 (62,8\%) ficaram internados por um período superior a 15 dias, o que difere de estudos realizados em unidades de clínica cirúrgica e de terapia intensiva, onde as médias de tempo de permanência foram 4 e 8 dias, respectivamente ${ }^{(6,20)}$. Apesar do tempo prolongado da internação, $67,3 \%$ dos pacientes receberam alta hospitalar, percentual também evidenciado em estudos no estado de São Paulo ${ }^{(12,21)}$, entretanto, é importante destacar que para 32,7\% o desfecho clínico da internação foi o óbito, taxa cinco vezes maior do que o encontrado em uma instituição hospitalar do município do Rio de Janeiro ${ }^{(18)}$. Em relação aos $11,5 \%$ dos pacientes que foram transferidos de setor no dia do incidente, questiona-se se este fato teria favorecido a ocorrência do erro, visto que as falhas na comunicação contribuem para a ocorrência de erros de medicação(22).

Quanto ao tipo de erro, 51,3\% estavam relacionados aos cinco certos da medicação (paciente, medicação, dose, via e horário certos) ${ }^{(5,23)}$, inferindo que a maioria dos erros ocorreu no preparo e administração. Os medicamentos não administrados representaram 28,3\%, que se assemelhou a outros estudos ${ }^{(2-3,5,7,12,17)}$.

Os erros relacionados à prescrição, transcrição e dispensação de medicamentos representaram 0,85\% dos incidentes. Chama atenção que em instituições que não possuem sistema de prescrição eletrônica, o percentual destes tipos de erros pode representar quase metade dos incidentes ${ }^{(22)}$.

A maioria dos incidentes, 60 (53,1\%) não causou nenhum dano ao paciente, porém em 53 (46,9\%) dos casos houve algum grau de dano, leve $29(25,7 \%)$, moderado $16(14,1 \%)$ ou grave oito $(7,1 \%)$, contrapondo estudo realizado em instituição pertencente à Rede Sentinela, em que ocorreu maior incidência de danos leves, e em contrapartida um óbito associado ao erro de medicação ${ }^{(6)}$. É importante destacar que a classificação do grau de dano é realizada pelo profissional que realiza as notificações, podendo ocorrer interpretações subjetivas. O near miss, por exemplo, correspondeu a 3,4\% das notificações, percentual muito divergente dos resultados de um estudo realizado em hospital público de São Paulo, no qual o quase erro representou $60,4 \%$ dos incidentes ${ }^{(24)}$.

A maior porcentagem de erros com danos ocorreu em pacientes com período de internação prolongada e hospitalizados em unidades fechadas, fato que pode ser explicado por tais unidades receberem pacientes mais graves e consequentemente, com maior quantidade e complexidade da terapia medicamentosa ${ }^{(5)}$. 
A ocorrência de erros de medicação associa-se mais frequentemente a atuação dos profissionais, como a não adesão a normas e protocolos ${ }^{(9)}$, falta de atenção ${ }^{(22)}$, falha na comunicação ${ }^{(2)}$, entre outros. Os erros e quase erros de medicação podem ser prejudiciais aos pacientes envolvidos, porém, as consequências destes incidentes também recaem sobre a equipe de enfermagem, como aumento da carga de trabalho ${ }^{(12)} \mathrm{e}$ necessidade de providências clínicas e administrativas ${ }^{(21-22)}$.

Visando a prevenção de novas ocorrências, as principais atividades de prevenção foram levantadas por um estudo multicêntrico, com base nas sugestões dos profissionais, sendo elas: aprimoramento das atitudes individuais dos profissionais, intensificação da educação continuada, reestruturação do quadro de pessoal, entre outras ${ }^{(22)}$.

A notificação foi realizada pelo enfermeiro em $99,1 \%$ dos casos, contrapondo estudo realizado em uma instituição semelhante, cujas notificações foram realizadas pela equipe multiprofissional ${ }^{(19)}$. Comparandose os índices de erros de medicação entre os hospitais gerais, com mais de 50 leitos, participantes do programa e grupos do CQH obteve-se mediana 0,11 e 0,08 nos meses de setembro/2014 e julho/2015, respectivamente, taxas que, variavelmente, se assemelham com as calculadas neste serviço. Em relação às quase falhas relacionadas ao processo de administração de medicação, as medianas foram 0,05 e 0,02 nos meses em questão, valores muito diferentes dos encontrados neste estudo, sugerindo a ocorrência de subnotificações ${ }^{(25)}$.

Frente à evidência de subnotificações, decorrentes do medo de sanções ético-legais possíveis, há necessidade de empenho para estimular as notificações, melhorar e desenvolver uma cultura para segurança. Considerar os erros de medicação como indicadores de resultado, pode contribuir para um novo olhar a esse tipo de evento. A valorização destes incidentes é fundamental, não só pela relevância numérica, mas principalmente, pelas consequências que podem causar ao paciente ${ }^{(12)}$.

Como limitação, este estudo apresentou a possibilidade de ocorrência de subnotificações. Importante ressaltar que o percentual de quase erro foi baixo.

\section{CONCLUSÃO}

A maioria dos pacientes internou pelo Sistema Único de Saúde e em unidades de internação clínicocirúrgicas, entretanto, houve maior quantidade de notificações de erros com danos em pacientes de Unidade de Terapia Intensiva e atendidos pela Saúde Suplementar.

Este estudo reitera a importância da notificação, mensuração e análise da ocorrência dos erros e quase erros de medicação, pois auxilia o enfermeiro a melhorar o processo de trabalho, na educação permanente do profissional de saúde e na prevenção e controle dos erros de medicação. Dos eventos ocorridos, 60 $(53,1 \%)$ não causaram danos ao paciente, 29 (25,7\%) causaram danos leves, $16(14,1 \%)$ moderados e oito $(7,1 \%)$ graves.

\section{- REFERÊNCIAS}

1. de Mello MC. Carga de trabalho em enfermagem: indicadores de tempo em unidades de clínica médica, cirúrgica e terapia intensiva adulto [tese]. São Paulo (SP): Universidade de São Paulo; 2011.

2. Yamamoto MS, Peterlini MAS, Boohomol E. Notificação espontânea de erros de medicação em hospital universitário pediátrico. Acta paul. enferm. [Internet] 2011;24(6) [acesso em 20 fev 2016]. Disponível: http://dx.doi.org/10.1590/S010321002011000600006.

3. Teixeira TCA, Cassiani SHB. Root cause analysis of falling accidents and medication errors in hospital. Acta paul. enferm. [Internet] 2014;27(2) [acesso em 20 fev 2016]. Disponível: http://dx.doi.org/10.1590/1982-0194201400019.

4. Compromisso com a qualidade hospitalar (CQH). Manual de indicadores de enfermagem NAGEH / Compromisso com a Qualidade Hospitalar (CQH). 2ª ed. São Paulo: APM/CREMESP; 2012.

5. Nascimento CCP, Toffoletto MC, Gonçalves LA, Freitas WG, Padilha KG. Indicadores de resultados da assistência: análise dos eventos adversos durante a internação hospitalar. Rev. Latino-Am Enfermagem. [Internet] 2008;16(4) [acesso 
em 20 fev 2016]. Disponível: http://dx.doi.org/10.1590/S0104-11692008000400015.

6. Paranaguá TTB, Bezerra ALQ, de Camargo e Silva AEB, de Azevedo Filho FM. Prevalência de incidentes sem dano e eventos adversos em uma clínica cirúrgica. Acta paul. enferm. [Internet] 2013;26(3) [acesso em 20 fev 2016]. Disponível: http://dx.doi.org/10.1590/S0103-21002013000300009.

7. Carneiro FS, Bezerra ALQ, de Camargo e Silva AEB, de Souza LP, Paranaguá TTB, Branquinho NCSS. Eventos adversos na clínica cirúrgica de um hospital universitário: instrumento de avaliação da qualidade. Rev. enferm. UERJ. [Internet] 2011;19(2) [acesso em 20 fev 2016]. Disponível: http://www.facenf.uerj.br/v19n2/v19n2a06.pdf.

8. Beccaria LM, Pereira RAM, Contrin LM, Lobo SMA, Trajano DHL. Eventos adversos na assistência de enfermagem em uma unidade de terapia intensiva. Rev Bras Ter Intensiva. [Internet] 2009;21(3) [acesso em 20 fev 2016]. Disponível: http:// www.scielo.br/pdf/rbti/v21n3/a07v21n3.pdf.

9. Mendes W, Pavão ALB, Martins M, Moura MLO, Travassos C. Características de eventos adversos evitáveis em hospitais do Rio de Janeiro. Rev. Assoc. Med. Bras. [Internet] 2013;59(5) [acesso em 20 fev 2016]. Disponível: http:// dx.doi.org/10.1016/j.ramb.2013.03.002.

10. Camerini FG, Colcher AP, Moraes DS, Souza DL, Vasconcelos JR, Neves RO. Fatores de risco para ocorrência de erro no preparo de medicamentos endovenosos: uma revisão integrativa. Cogitare Enferm. [Internet] 2014;19(2) [acesso em 20 fev 2016]. Disponível: http://dx.doi.org/10.5380/ce.v19i2.37362.

11. dos Santos JC, Ceolim MF. latrogenias de enfermagem em pacientes idosos hospitalizados. Rev. esc. enferm. USP. [Internet] 2009;43(4) [acesso em 20 fev 2016]. Disponível: http://dx.doi.org/10.1590/S0080-62342009000400011.

12. Toffoletto MC, Padilha KG. Consequências de medicações em unidade de terapia intensiva e semi-intensiva. Rev. esc. enferm. USP. [Internet] 2006;40(2) [acesso em 20 fev 2016]. Disponível: http://dx.doi.org/10.1590/S008062342006000200013.

13. Organização Mundial da Saúde (OMS). Relatório técnico. Estrutura concetual da classificação internacional sobre segurança do doente [Internet] Lisboa: Direção-Geral da Saúde; 2011 [acesso em 20 fev 2016]. Disponível: http://apps. who.int/iris/bitstream/10665/70882/4/WHO_IER_PSP_2010.2_por.pdf?ua=1.

14. de Vries EN, Ramrattan MA, Smorenburg SM, Gouma DJ, Boermeester MA. The incidence and nature of in-hospital adverse events: a systematic review. Qual Saf Health Care. [Internet] 2008;17(3) [acesso em 20 fev 2016]. Disponível: http://dx.doi.org/10.1136/qshc.2007.023622.

15. World Health Organization (WHO). Patient safety: rapid assessment methods for estimating hazards. Report of the WHO working group meeting. [Internet] Geneva: WHO; 2003 [acesso em 20 fev 2016]. Disponível: http://www.who.int/ patientsafety/activities/system/en/rapid_assessment_methods.pdf.

16. Mendes W, Martins M, Rozenfeld S, Travassos C. The assessment of adverse events in hospitals in Brazil. Int J Qual Health Care. [Internet] 2009;21(4) [acesso em 20 fev 2016]. Disponível: http://dx.doi.org/10.1093/intghc/mzp022.

17. Barker KN, Flynn EA, Pepper GA, Bates DW, Mikeal RL. Medication errors observed in 36 health care facilities. Arch Intern Med. [Internet] 2002;162(16) [acesso em 20 fev 2016]. Disponível: http://archinte.jamanetwork.com/article. aspx?articleid=212740.

18. Roque KE, Melo ECP. Tempo de internação e a ocorrência de eventos adversos a medicamentos: uma questão da enfermagem. Esc. Anna Nery. [Internet] 2011;15(3) [acesso em 20 fev 2016]. Disponível: http://dx.doi.org/10.1590/S141481452011000300022.

19. Bezerra ALQ, de Camargo e Silva AEB, Branquinho NCSS, Paranaguá TTB. Análise de queixas técnicas e eventos adversos notificados em um Hospital Sentinela. Rev. enferm. UERJ. [Internet] 2009;17(4) [acesso em 20 fev 2016]. Disponível: http://www.facenf.uerj.br/v17n4/v17n4a02.pdf.

20. Reis WCT, Scopel CT, Correr CJ, Andrzejevski VMS. Análise das intervenções de farmacêuticos clínicos em um hospital de ensino terciário do Brasil. Einstein. [Internet] 2013;11(2) [acesso em 20 fev 2016]. Disponível: http://dx.doi. org/10.1590/S1679-45082013000200010.

21. de Carvalho VT, Cassiani SHB. Erros na medicação e conseqüências para profissionais de enfermagem e clientes: um estudo exploratório. Rev. Latino-Am Enfermagem. [Internet] 2002;10(4) [acesso em 20 fev 2016]. Disponível: http://dx.doi. org/10.1590/S0104-11692002000400009. 
22. Miasso Al, Grou CR, Cassiani SHB, de Camargo e Silva AEB, Fakih FT. Erros de medicação: tipos, fatores causais e providências tomadas em quatro hospitais brasileiros. Rev. esc. enferm. USP. [Internet] 2006;40(4) [acesso em 20 fev 2016]. Disponível: http://dx.doi.org/10.1590/S0080-62342006000400011.

23. Teixeira TCA, Cassiani SHB. Análise de causa raiz: avaliação de erros de medicação em um Hospital Univeristário. Rev. esc. enferm. USP. [Internet] 2010;44(1) [acesso em 20 fev 2016]. Disponível: http://dx.doi.org/10.1590/S008062342010000100020 .

24. D'Aquino FFR. Incidentes relacionados a medicamentos em uma instituição hospitalar: subsídios para a gestão [tese]. Botucatu (SP): Universidade Estadual Paulista; 2014.

25. Compromisso com a qualidade hospitalar $(\mathrm{CQH})$. Indicadores hospitalares. [Internet] Estatísticas. Comparativo de indicadores dos Hospitais Participantes do programa de grupos do CQH [acesso em 20 fev 2016]. Disponível: http:// www.cqh.org.br/icqh/estat/ind/web.php. 